\title{
THEORETICAL AND EXPERIMENTAL INVESTIGATIONS TO DEFINE OPTIMAL PARAMETERS OF THE STRAIGHT-FLOW TURBINE FOR NON-DAM HYDRO POWER STATION
}

\author{
Dinara TURALINA ${ }^{1}$, Daniyar BOSSINOV ${ }^{* 2}$ \\ 1,2 Dept. of Mechanics, Kazakh National University named after Al-Farabi, Al-Farabi 71, Almaty, \\ Kazakhstan \\ *d.bossinov@ @atbayev.university \\ Received: 12 February 2018; Accepted: 25 April 2018
}

In the article, there are theoretical and experimental investigations presented with regards to determination of optimal parameters of the straight-flow turbine for non-dam hydro power stations. The goal for conducting theoretical and experimental investigations is to increase electric power of the hydro turbine. The most optimal angle of attack of the flow direction at the inlet has been calculated. Accordingly, the results of velocity, pressure, lift and drag forces as well as lift and drag coefficients along the blade have been received. Also, hydro turbine's work with nonrotating and rotating shaft modes have been taken into account. The achieved results executed in $2 D$ and $3 D$ simulation software modes allowed visualization of the hydro turbine performance through depicting arrows, streamlines, contours, surfaces and line graphs. The calculations with regards to incompressible liquids were measured with the help of COMSOL Multiphysics and ANSYS Fluent software packages through application of Direct Numerical Simulation (DNS) and K-epsilon methods on the base of Navier-Stokes equations. Changing water discharge through a ball valve, the amount of the rotation of the hydraulic turbine rotor was determined using a tachometer. The produced electric power due to the rotation of the hydraulic turbine rotor in minutes is calculated according to Ohm's law.

Key words: Renewable Energy Sources (Renewables), Hydro Turbine, Navier-Stokes Equations, Comsol Multiphysics, Ansys Fluent

\section{Introduction}

Reduction of costs for electricity production is one of the key issues of the current day. The price of solid, liquid and gaseous fuels used in traditional power plants for heating is increasing day by day, and its environmental impact is high. Meanwhile, the invested huge sums to ensure environmental security of those plants do not contribute to their efficiency unless they switch to alternative sources of energy. It is therefore beneficial to use renewable energy sources where a nondam hydro power station with a straight-flow turbine could serve a good example. A key benefit of this hydro turbine is its small volume that means less material for its production is needed and 
consequently the price appears to be much cheaper. Another advantage is that it does not require any dams that means a significant reduction of costs allocated for dam construction and minimization of impacts on the ecosystems of the river, which is not limited in its currents (flow).

\section{The concept of hydro turbine}

Hydro turbine is a hydraulic engine that transforms the energy of the current (flow) into mechanical energy with the help of the hydraulic turbine rotor. Through the rotor, this engine is connected to the hydraulic turbine generator and the compounds altogether form a hydraulic unit. Thus, hydraulic energy is converted to mechanical energy in the hydro turbine and then inside the hydraulic turbine generator is transformed to electrical energy $[2,7]$.

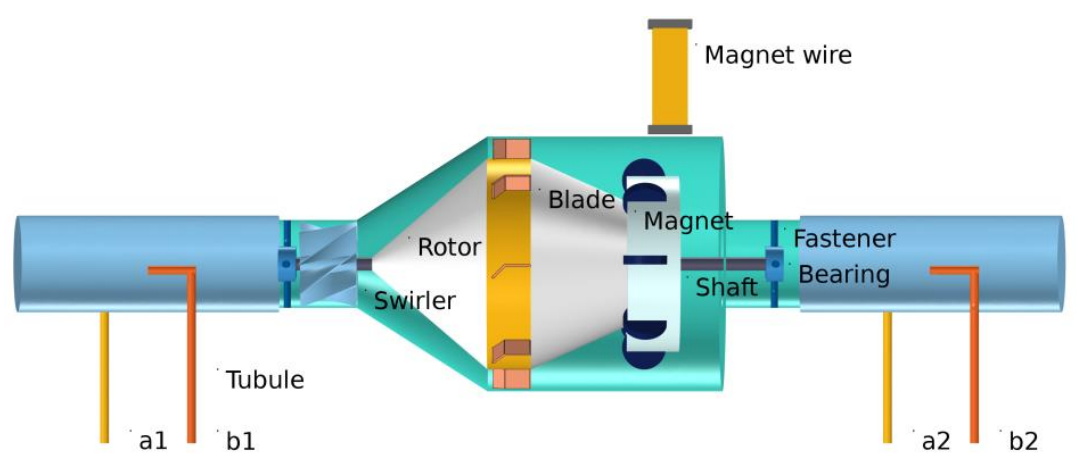

Figure 1. A transparent view of the flow turbine

Hydro turbine works as follows. The water flows from the left pipe and moves through the water swirler and circulates around the rotor. The water flow affects the blades and contributes to appearance of the lifting power, which turns the rotor along its axis. Due to the rotation of magnets, the hydraulic unit produces electricity in its magnet wire. The water running through the hydro turbine goes out via the right pipe (Fig.1 and Fig.22).

\section{Defining the most effective parameters of the blade}

\section{Mathematical model of the 2D fluid domain}

The fluid in which the blade will be deployed is an ideal incompressible Newtonian viscous flow. The basic equations are considered for the physical system [3-7]:

Conservation of mass (continuity)

$$
\nabla \cdot u=0
$$

Conservation of momentum (Newton's second law)

$$
F=m a
$$

For a fluid particle, it is convenient to divide Eq. (2) by the volume of the particle to provide possibility to work with density instead of mass, as it is shown in Eq. (3). 


$$
\rho \frac{D u}{D t}=\rho \frac{\partial u}{\partial t}+\rho(u \cdot \nabla) u=\rho g+F \text { in } \Omega \text { for } t \in[0, T]
$$

Where $\rho g$ is the applied body force per unit volume on the fluid particle, $\Omega$ is the domain occupied by the fluid at time $t, F$ is the surface force which is applied by external stress on the sides of the element, for Newtonian fluid,

$$
F=\nabla \cdot \sigma
$$

in which $\sigma$ is the total stress tensor:

$$
\sigma=-p I+\mu\left(\nabla u+(\nabla u)^{T}\right)
$$

Combining Eq.(3), (4) and (5), the Navier-Stokes Equation for a Newtonian fluid consisting of pressure and viscous stress is given in Eq. (6)

$$
\rho \frac{\partial u}{\partial t}+\rho(u \cdot \nabla) u=\nabla \cdot\left[-p I+\mu\left(\nabla u+(\nabla u)^{T}\right)\right]+\rho g
$$

An application mode based on this equation is available in COMSOL Multiphysics in the fluid module to model the ideal incompressible Newtonian flow.

When solving the task in the software COMSOL Multiphysics, a blade part located in the hydro turbine rotor was selected for calculations (Fig.2).

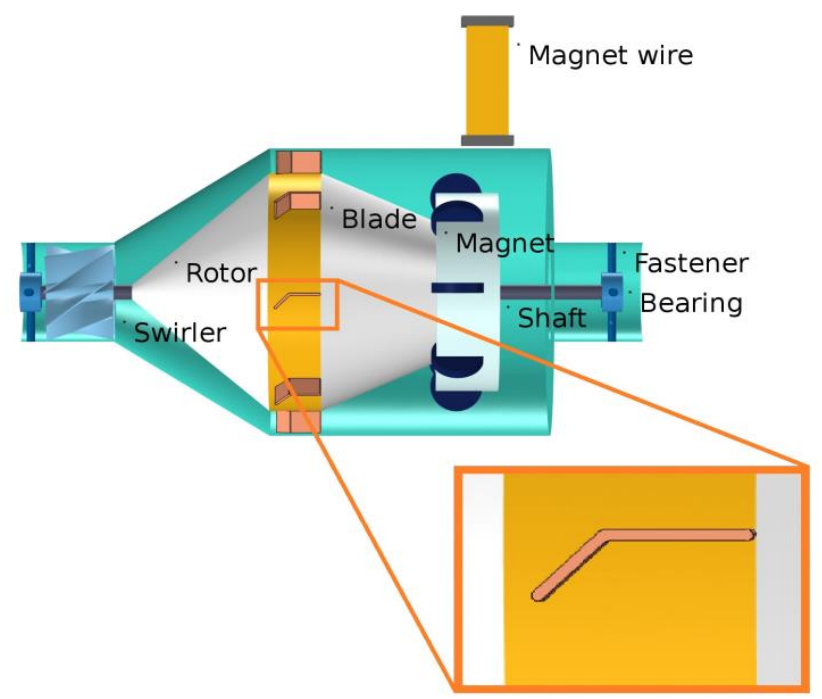

Figure 2. The part of the blade is located in the hydro turbine rotor

To solve the two-dimensional task numerically with the help of the software COMSOL Multiphysics, a part of the blade located in the turbine rotor was chosen (Fig.3). The mesh allocation in two-dimensional domain is illustrated in Fig.4. 


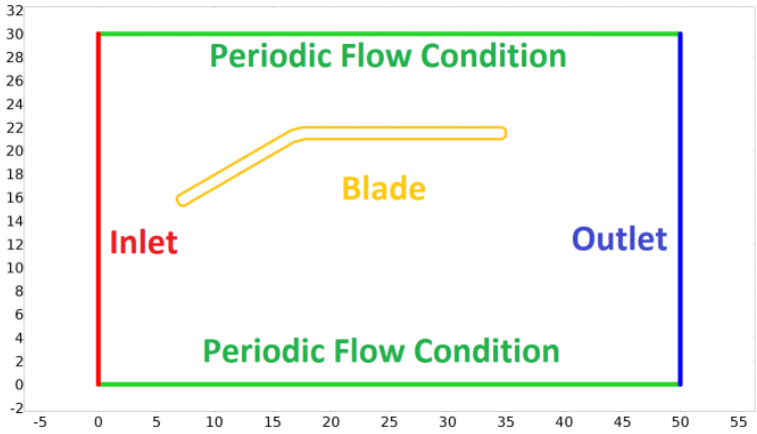

Figure 3. Boundary conditions

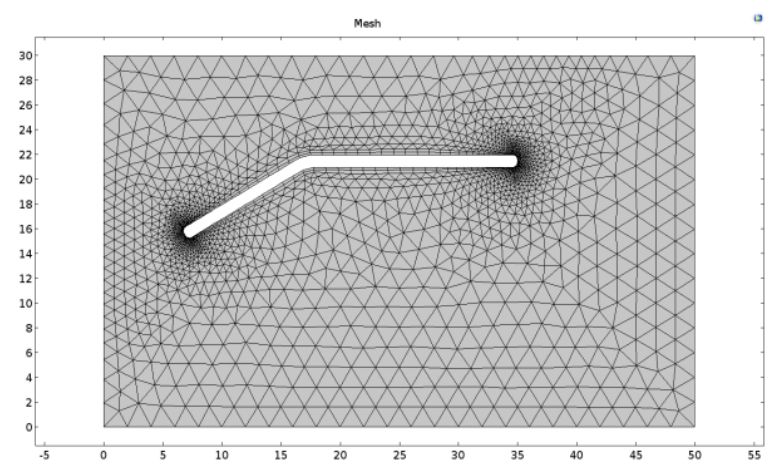

Figure 4. Mesh allocation in 2D domain

Boundary conditions:

Inlet:

\begin{tabular}{|l|l|}
\hline $\mathrm{x}$ & $U \cdot \cos (\alpha \cdot \pi / 180)$ \\
\hline $\mathrm{y}$ & $U \cdot \sin (\alpha \cdot \pi / 180)$ \\
\hline
\end{tabular}

Outlet: Neumann boundary condition

Periodic flow condition: $u_{\text {source }}=u_{\text {dest },} p_{\text {source }}=p_{\text {dest }}$

Wall: No slip

Fluid properties are shown in the Tab.1.

Table 1. Fluid properties

\begin{tabular}{|c|c|c|c|}
\hline $\begin{array}{c}\text { Dynamic } \\
\text { viscosity }(\mathrm{Pa} \cdot \mathrm{s})\end{array}$ & $\begin{array}{c}\text { Kinematic viscosity } \\
\mathrm{m}^{2} / \mathrm{s}\end{array}$ & $\begin{array}{c}\text { Density } \\
\mathrm{kg} / \mathrm{m}^{3}\end{array}$ & $\begin{array}{c}\text { Temperature } \\
\mathrm{C}^{0}\end{array}$ \\
\hline 0.001 & $1 \cdot 10^{-6}$ & 999 & 20 \\
\hline
\end{tabular}

There are two ways to change the angle of attack of the model. It is possible either to rotate the blade itself [2] or to keep the blade fixed but modifying the flow direction at the inlet. In the provided experiment the second approach is applied. It is much simpler to adjust the velocity field at the inlet boundary condition as in this case there is no need to remesh the model for each angle of attack. As shown in the Fig.5 and Fig.6, the blade is fixed while the arrows show the flow at an angle of attack due to the adjusted inlet velocity direction.

The initial velocity field of the water in $\mathrm{x}$ and $\mathrm{y}$ directions are defined as $U \cdot \cos (\alpha \cdot \pi / 180)$ and $U \cdot \sin (\alpha \cdot \pi / 180)$ respectively. Here $U=1 \mathrm{~m} / \mathrm{s}$, and values of $\alpha$ are $0^{0}, 15^{0}, 30^{0}, 45^{0}, 60^{0}, 75^{0}, 90^{0}$. By changing the direction of the water, the highest value of the lift force along the blade is selected. Accordingly, the optimal angle of the swirler is determined.

The boundary conditions are set up in Periodic Flow Condition due to the fact that the chosen blade is affected by the changes in the water velocity running along the lower and upper blades. The 
length of the rectangular channel is $L=30 \mathrm{~mm}$ and width $W=50 \mathrm{~mm}$. The length of the blade is 28.25 $\mathrm{mm}$ and its width constitutes $2 \mathrm{~mm}$.

The task is defined as time dependent, and after 10 seconds computations calculated the figures on velocity, pressure, lift and drag forces as well as lift and drag coefficients. Changes in water velocity from the dark blue to the dark red are shown in the right column of the bar. The dark blue color of water signals vortex and cavitation, while dark red color of water shows a significant increase of velocity. When it comes to the distribution of the pressure, the altering color of water from dark blue to dark red witnesses a significantly increased pressure.

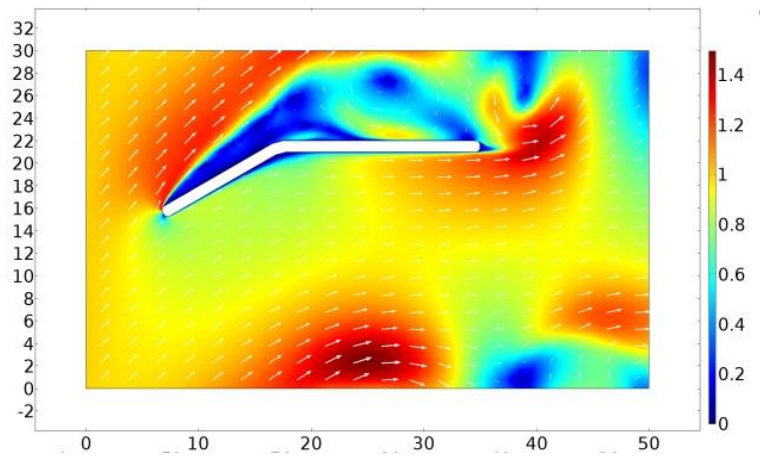

Figure 5. Velocity magnitude $(\mathrm{m} / \mathrm{s})$

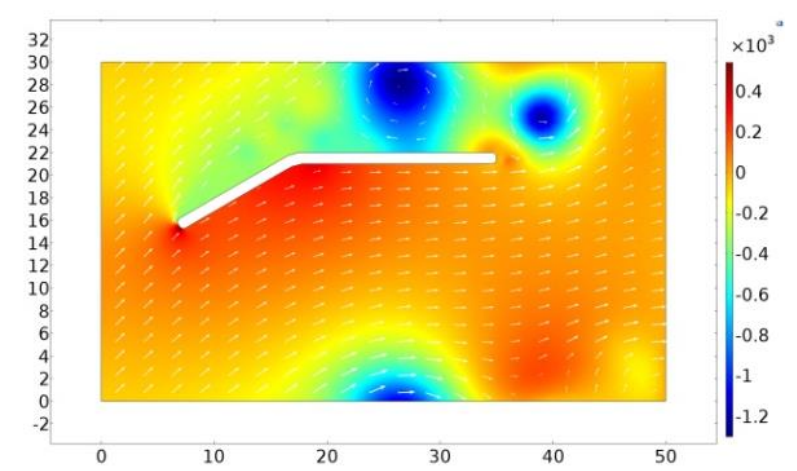

Figure 6. Pressure (Pa)

Fig. 5 shows simulation of flow passing the blade at a 45 -degree angle of attack. The surface plot illustrates the velocity magnitude along with the arrows (shown in white). In Fig.6 pressure is shown along the blade. Values of pressure increase in the bottom side of the blade as a result of the appearing lift force.

\section{Calculation of the lift and drag forces}

When fluid flow passes a body, it exerts a force on the surface. As shown in the figures below, the force component that is perpendicular to the flow direction is called Lift. The force component that is parallel to the flow direction is called Drag. Fig.7 and Fig.8 provide respective schematic illustrations on lift and drag forces

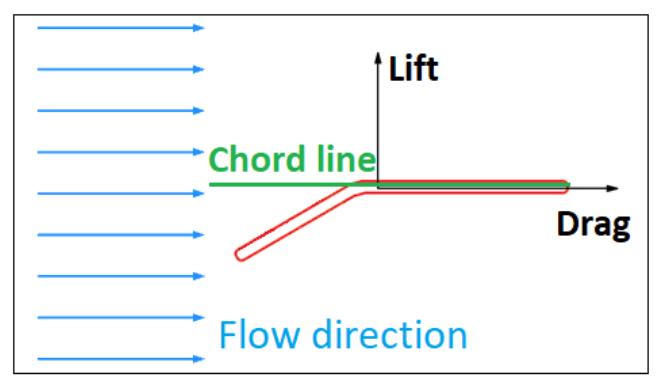

Figure 7. Flow at a 0 -degree angle of attack

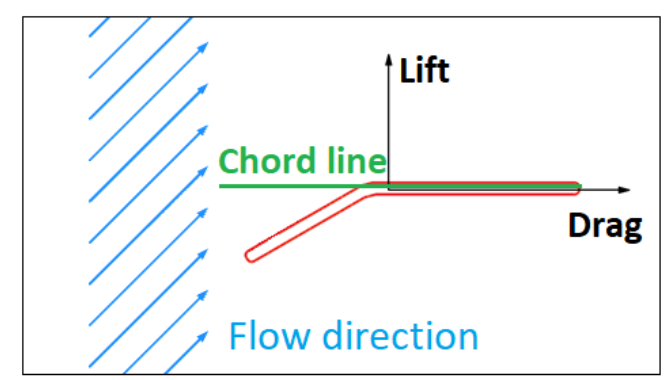

Figure 8. Flow at a 45-degree angle of attack 
It is common that the geometry may not be aligned perfectly with the flow direction. The angle between the center reference line of the geometry and the incoming flow is called angle of attack. In aerospace engineering, the angle of attack is frequently used as it is the angle between the chord line of the airfoil and the free-stream direction.

There are two distinct contributors to lift and drag forces - pressure force and viscous force. The pressure force, often referred to as pressure-gradient force, is the force appearing due to the pressure difference across the surface. The viscous force is the force deriving from friction that acts in the opposite direction of the flow. For 2D fluid flow, the drag force and lift force are given [3] by the Eq.7 and Eq.8 respectively:

$$
\begin{gathered}
F_{D}=\int_{S}\left(\rho_{f} \mu_{f} \frac{\partial u}{\partial n} n_{y}-p n_{x}\right) d S \\
F_{L}=\int_{S}\left(\rho_{f} \mu_{f} \frac{\partial u}{\partial n} n_{x}+p n_{y}\right) d S
\end{gathered}
$$

Fig.9 and Fig.10 illustrate graphs of lift and drag forces' changes indicated within 10 seconds period due to the changing water direction. The frequency and amplitude of oscillations in the figures below happen due to perturbations in the flow caused by the vortex.

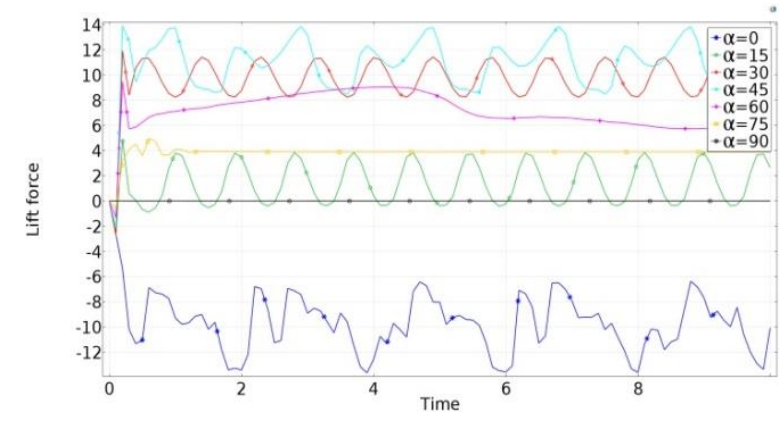

Figure 9. Lift forces at various angle of flow attack

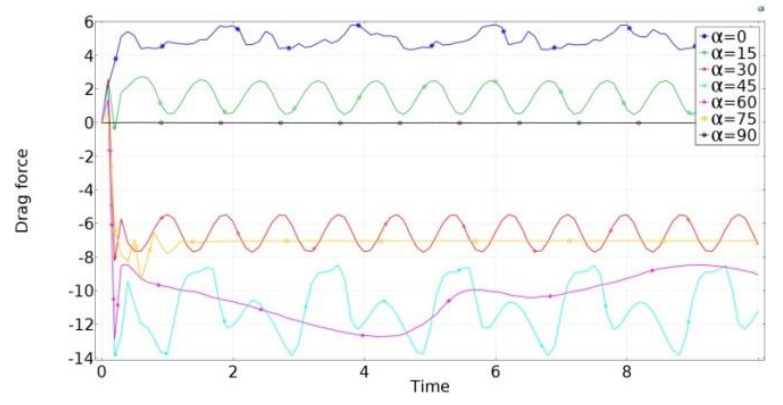

Figure 10. Drag forces at various angle of flow attack

During the calculations the lift force maximum value was indicated when the water flow direction was 45 degrees. When the angle of attack equals 0 and 90 degrees, there is no lift force as the water does not affect the bottom side of the blade. Between 75 and 90 degrees a stall happens due to the reduction of the lift force generated by the blade as angle of attack increases. This occurs when the critical angle of attack of the blade is exceeded.

In Fig.10 the maximum value of the Drag force achieves in the 0 -degree angle of attack. Similarly like in Fig.9, in this case at 90-degree angle of attack, the value of the drag force is also zero. This happens due to the absence of the flow velocity in the domain. 


\section{Calculation of the lift and drag coefficients}

The expression for the drag and lift coefficient in terms of drag force and lift force for 2D flow is given [3] by the Eq.(9) and Eq.(10)

$$
\begin{gathered}
C_{D}=\frac{2 F_{D}}{\rho f u^{2} d} \\
C_{L}=\frac{2 F_{L}}{\rho f u^{2} d}
\end{gathered}
$$

It is often easier to use the coefficients instead of the dimensional forces for the purpose of validating experimental data or comparing different designs. In our case the nondimensionalized forms of the lift and drag coefficients defined as:

$$
\begin{aligned}
& C_{L}(\alpha)=\phi_{c}\left(c_{p}(s) / c\right)\left(n_{y}(s) \cos (\alpha)-n_{x}(s) \sin (\alpha)\right) d s \\
& C_{D}(\alpha)=-\phi_{c}\left(c_{p}(s) / c\right)\left(n_{y}(s) \cos (\alpha)-n_{x}(s) \sin (\alpha)\right) d s
\end{aligned}
$$

where the pressure coefficient defined as:

$$
c_{p}(s)=\frac{p(s)-p}{\frac{1}{2} \rho U^{2}}
$$

- $c$ is the blade length

- $p$ is the fluid's pressure

- $\rho$ is the fluid's density

- $U$ is the mean velocity

- $n_{x}$ is the unit normal vector in the $\mathrm{x}$ direction

- $n_{y}$ is the unit normal vector in the y direction

- $s$ is the contour of the blade

- $\alpha$ is the angle of attack in degrees.

- $C_{L}$ is the dimensionless lift coefficient

- $C_{D}$ is the dimensionless drag coefficient

Fig.11 and Fig.12 show dimensionless lift and drag coefficients at various angles of attack during ten seconds.

Lift and drag coefficients vary from -1 to 1 . 


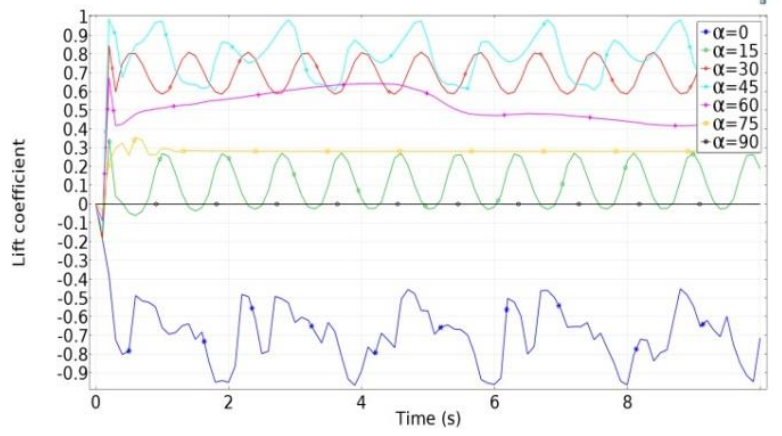

Figure 11. Dimensionless lift coefficient at a various angle of attack of flow

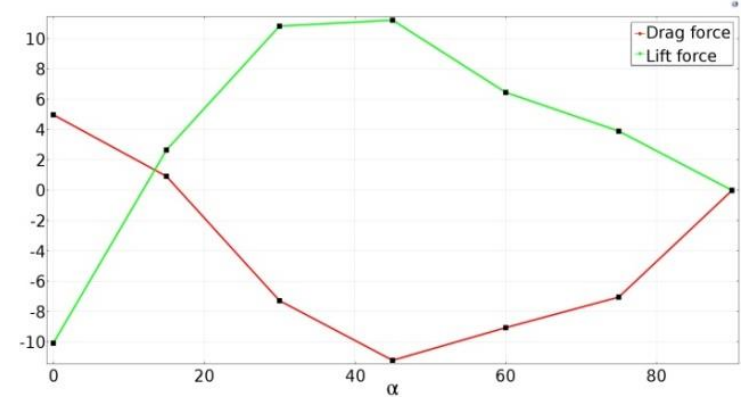

Figure 13. Lift and drag forces at various angle of attack in the tenth second

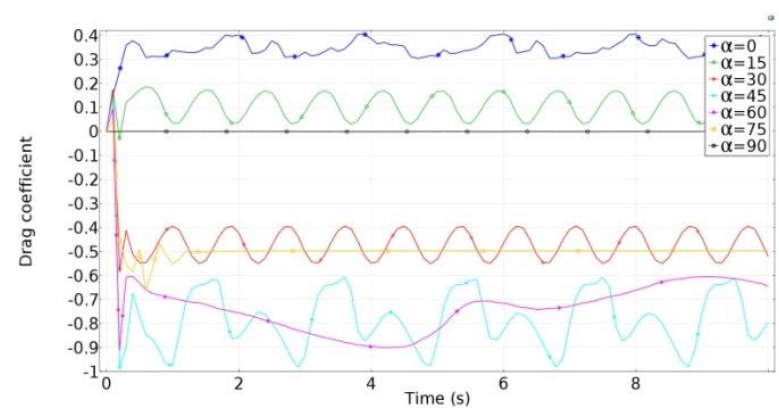

Fig.12. Dimensionless drag coefficients at a various angle of attack of flow

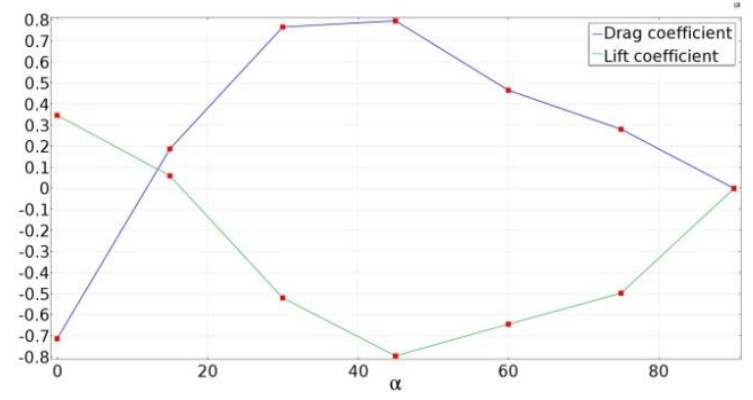

Figure 14. Lift and drag coefficients at various angle of attack in the tenth second

Lift, drag forces and coefficients at various angles of attack in the tenth second are shown in Fig.13 and Fig.14. The black and red points mean data in each degree.

\section{Visualization of hyrdro turbine performance}

Numerical calculations on the three-dimensional module of the hydro turbine performance allow us to observe how the flow of water affects the blades and rotates the rotor of the hydro turbine. The three-dimensional module of the hydro turbine makes the processes more realistic and feasible in comparison with its counterpart with two-dimensional projection.

The properties of the liquid in both two-dimensional and three-dimensional cases are the same.

In the three-dimensional view, the hydro turbine rotor has been accounted in rotating and nonrotating modes in ANSYS Fluent software package.

When the water flow direction equals to 45 degrees, the values of the lift and the drag forces appear to increase and decrease for maximum, respectively (Fig.9 and Fig.10). In this regard, the swirler directions are fixed as 45 degrees and the angle of flow attack runs to the blades at the same degree value. Accounting the described situation, it has been found that the most effective flow direction to the blade in the water spray pipe is 45 degrees.

The Case of Non-rotating Rotor Mode 
The mathematical model for non-rotating hydro turbine rotor was the same like in case of the two-dimensional mode. The initial water velocity constituted $0.1 \mathrm{~m} / \mathrm{s}$ for this mode. For better visualization purpose the difference in water speed has been shown with the help of Rainbow color option. The range of change in water velocity from blue to red is shown in the left column of the Fig.15 and Fig.16.

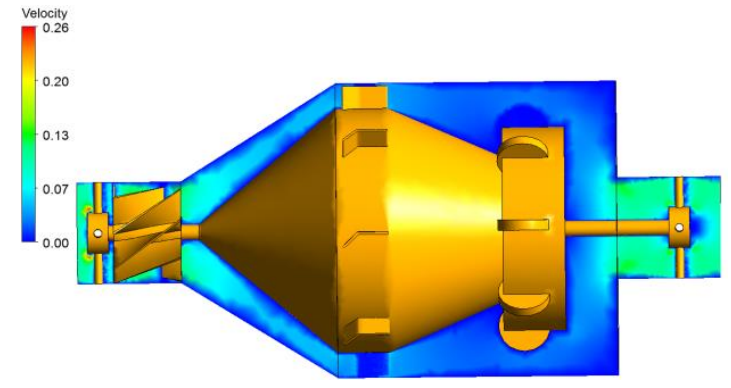

Figure 15. Velocity surface $(\mathrm{m} / \mathrm{s})$

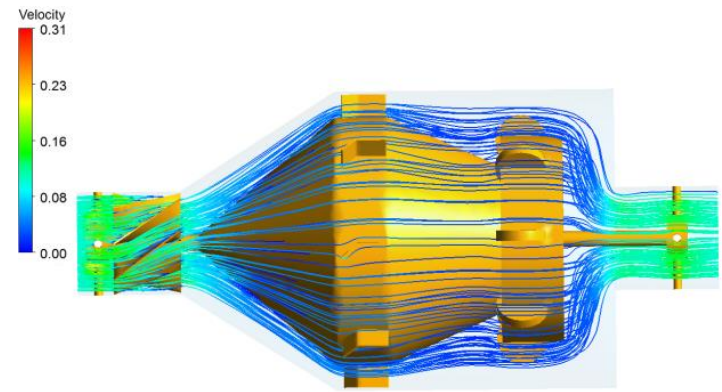

Figure 16. Velocity streamlines $(\mathrm{m} / \mathrm{s})$

The turn of the water color to blue indicates a maximum decrease in velocity, and the red color in opposite shows that velocity increases for maximum. Velocity streamlines around the swirler and rotor illustrate water flow directions in Fig.16.

\section{Case of Rotating Rotor Mode}

All calculations have been undertaken in the software ANSYS Fluent with the help of Mesh Motion, k-epsilon model. To simplify the task, the shaft has been excluded. The water velocity at Inlet is $0.8 \mathrm{~m} / \mathrm{s}$. Rotating domain revolves 155 times per minute along the axis $z$ (as the eighth experiment in Tab.2).

In Fig.17 and Fig.18, the flow direction is shown with streamlines. Along the rotor the streamlines undergo certain changes running through circulation, which intensifies towards the end of the rotor turning to a vortex. In turn, vortex stimulates the process of cavitation that damages the entire hydro turbine surface. The colors of streamlines mean velocity value.
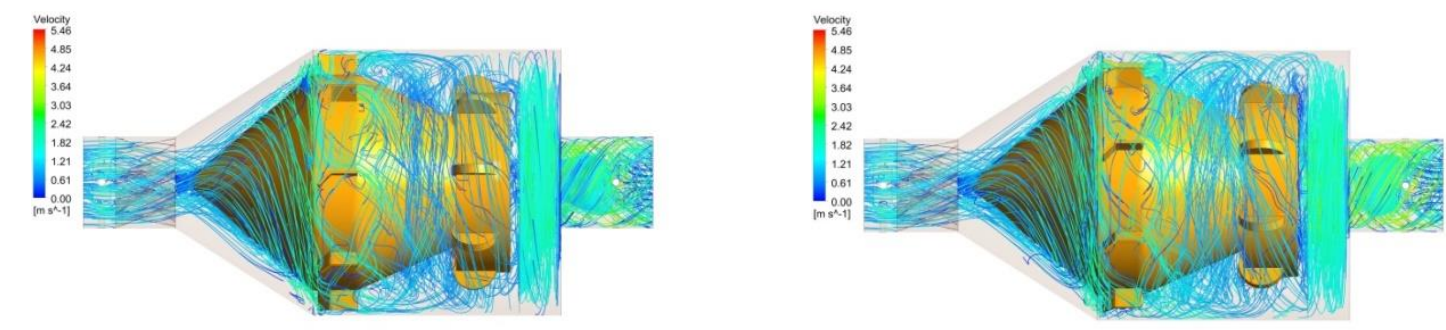

\section{Figure 17. Velocity streamlines at $t=0.5 \mathrm{~s} \quad$ Figure 18. Velocity streamlines at $t=2.5 \mathrm{~s}$}

Water velocity reaches its maximum value in the hydro turbine part where the blades are located and at the end of the rotor (Fig.19). 

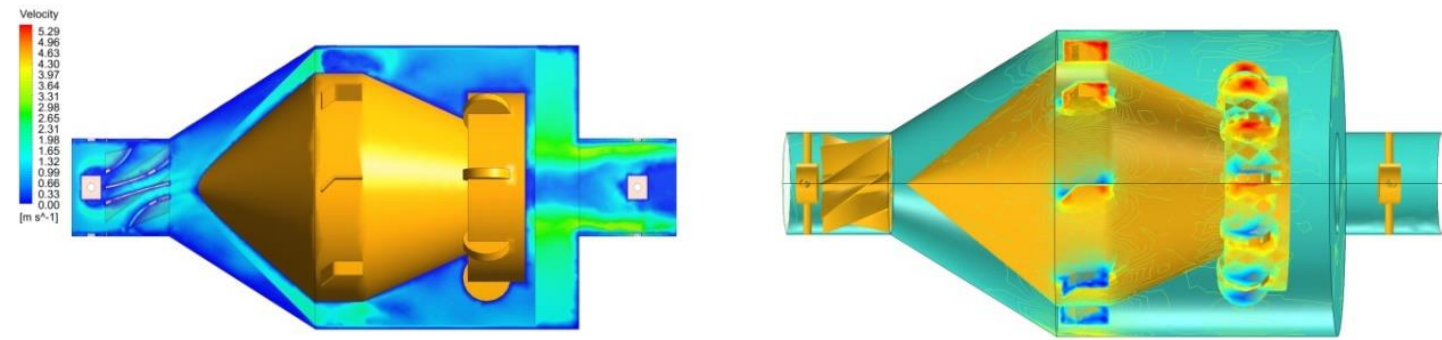

Figure 19. Velocity surface at $t=0.5 \mathrm{~s}(\mathrm{~m} / \mathrm{s})$

Figure 20. Pressure contour $(\mathbf{P a})$

As illustrated in Fig.20, vortex appears in the upper side of the blade due to subatmospheric (negative) pressure, whereas positive pressure takes place when the water flow pushes the bottom side of the blade. In this figure, the positive pressure is shown with red color, while the negative one is dyed in blue.

Lift force appears due to the pressure difference between the upper and lower surfaces of the blade. The lifting force pushes the lower surface of the blade due to which the hydro turbine rotor revolves.

\section{Experimental researches}

The Fig.21 and Fig.2 illustrate the general view of the straight-flow hydro turbine and one applied during experimental tests [2].

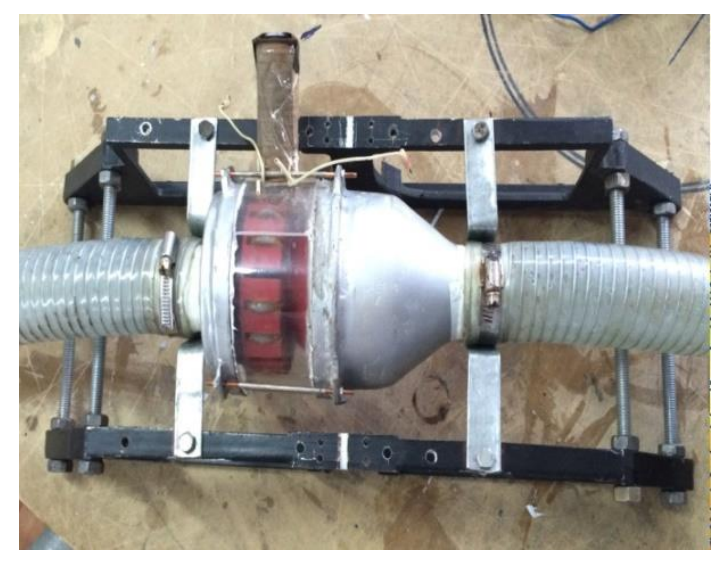

Figure 21. A general view of the straight-

\section{flow turbine}

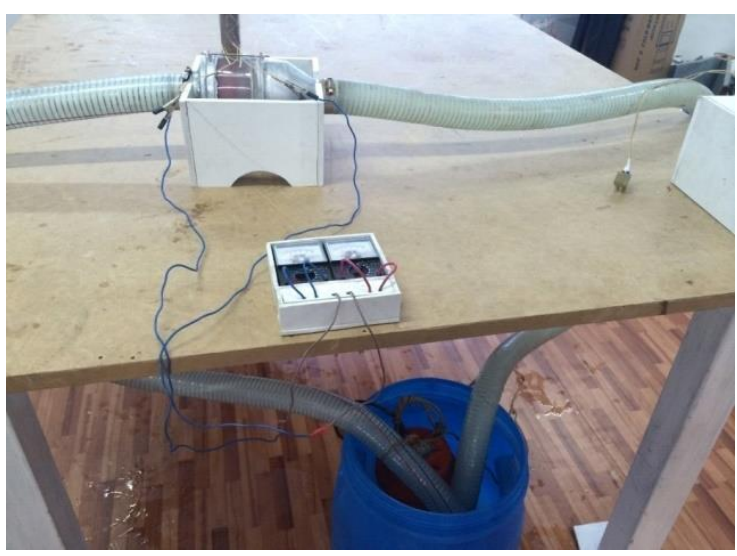

Figure 22. The experimental device applied during the studies

The experimental device comprises pipes, a reservoir, a straight-flow hydro turbine, a magnet wire, a pump, a ball valve changing the water head and water discharge, a tachometer calculating the rotation of the hydro turbine rotor, an ammeter measuring the electricity, and a multimeter applied to measure voltage [1, 2] (Fig.22).

The outcomes of the experimental studies run in the straight-flow hydro turbine are presented in the Tab. 2 below: 
Table 2. Hydro turbine values regarding water discharge

\begin{tabular}{|l|l|l|l|l|}
\hline № & $\begin{array}{c}\text { Water } \\
\text { discharge } \\
\mathrm{m}^{3} / \mathrm{s}\end{array}$ & $\begin{array}{c}\text { Flow velocity } \\
\mathrm{m} / \mathrm{s}\end{array}$ & $\begin{array}{c}\text { Hydro turbine } \\
\text { rotor } \\
\text { revolution per } \\
\text { minute }\end{array}$ & $\begin{array}{c}\text { Electricity } \\
\text { production } \\
W t\end{array}$ \\
\hline 1 & 0.0013 & 0.17 & 28 & 0.5 \\
\hline 2 & 0.0018 & 0.23 & 55 & 0.7 \\
\hline 3 & 0.0022 & 0.28 & 68 & 1.4 \\
\hline 4 & 0.0028 & 0.36 & 80 & 4.5 \\
\hline 5 & 0.003 & 0.38 & 95 & 5 \\
\hline 6 & 0.0042 & 0.535 & 120 & 6.2 \\
\hline 7 & 0.005 & 0.64 & 150 & 8 \\
\hline 8 & 0.0062 & 0.8 & 155 & 8.1 \\
\hline
\end{tabular}

As the experimental study shows, the straight-flow hydro turbine operates within the entire alteration of the water discharge. The pump sucks up water from the water reservoir and delivers it to the hydro turbine through the pipe. With the help of the water flow, the hydro turbine rotor rotates and the passed water comes to the reservoir through the pipe back. Changing water discharge through a ball valve, the amount of the rotation of the hydraulic turbine rotor was determined using a tachometer. The produced electric power due to the rotation of the hydraulic turbine rotor in minutes is calculated according to Ohm's law [1].

\section{Conclusions}

Numerical modeling allows calculation of the most optimal parameters of a hydro turbine. In turn, the numerical calculations provide an opportunity to decrease financial costs on materials to manufacture prototypes (experimental templates) of a hydro turbine. At the same time, the conducted 2D and 3D modeling of the hydro turbine calculations is applicable to verify the capacity of the designed construction (a prototype). Furthermore, the undertaken scope of experimental works builds grounds to solve a number of other tasks of fluid mechanics (for example, to define lift force of jet wings, wind turbine blades and etc.).

The presented construction is especially useful for tropic climate areas where water does not experience freezing effect. In this case, the constructed devices can operate throughout the year.

Finally, the construction is in compliance with the contemporary world trends appreciating pro-environmental behavior as it does not require damaging ecosystems through building dams, for instance.

During the world exhibition "Expo-2017: Future Energy" (Astana, Kazakhstan, June 10 September 10, 2017) the visitors witnessed a straight-flow non-dam hydro turbine presented as an exposition.

\section{Acknowledgements}

The authors acknowledge the support provided by Committee of Science of the Ministry of Education and Science of the Republic of Kazakhstan, under the grant funding (AP05130503) named "Management of energy-saving modes of oil transportation through main oil pipelines using the SCADA system". 


\section{References}

[1] D. Zh. Bossinov, M.B Koshumbayev. Innovative patent «Hydraulic unit» - № 31166 16.05.2016.

[2] D. E. Turalina, D. Zh. Bossinov. «Theoretical and experimental investigations to define optimal parameters of the straight-flow turbine for non-dam hydro power station»// VESTNIK KazNU. (Series Mathematics, Mechanics, Informatics). 2015. - №1 (84). — p. 124 - 131.

[3] Jamnani Dinesh. "Modeling and Simulation of a Single Particle in Laminar Flow Regime of a Newtonian Liquid", COMSOL conference, Bangalore (2009)

[4] R.Han, J.Cherry and R. Kallernberg. Modeling an Oscillating Water Foil for Hydro-kinetic Power Generator Using COMSOL 3.5a. Excerpt from the Proceedings of the COMSOL Conference 2010 Boston.

[5] Loytsyanskii L.G. Mechanics of liquids and gases. - 6th Edition, Moscow: Science, 1987.$840 \mathrm{p}$.

[6] Turbulent Flows by Stephen B. Pope. Cambridge University Press: Cornell University, 2000. $-773 \mathrm{p}$.

[7] Naixing Chen. AEROTHERMODYNAMICS OF TURBOMACHINERY, ANALYSIS AND DESIGN. Institute of Engineering Thermophysics, Chinese Academy of Sciences, China, 2010. $-461 \mathrm{p}$. 